\section{Toleranzaufbau bei Erdnussallergie}

\author{
Wie wirksam eine orale Immuntherapie bei Kindern mit \\ Allergie gegen Erdnussprotein ist, war die Fragestellung einer \\ britisch-amerikanischen Studie.
}

nsgesamt 39 Kinder im Alter von ein bis neun Jahren erhielten eine orale Immuntherapie mit Erdnussprotein. Am ersten Tag erfolgte stationär, beginnend mit $0,1 \mathrm{mg}$ Erdnussprotein, die erste Dosissteigerung. Die Dosis wurde alle 30 Minuten bis maximal $50 \mathrm{mg}$ verdoppelt. 26\% der Kinder erreichten diese Höchstdosis, 38\% tolerierten $25 \mathrm{mg}$.

Die höchste tolerierte Dosis war die Startdosis der folgenden Aufbauphase. Dabei wurden Dosissteigerungen von $25 \mathrm{mg}$ alle zwei Wochen bis zu einer Maximaldosis von $300 \mathrm{mg}$ vorgenommen, wobei die Dosiserhöhung jeweils in der Klinik und die weitere Einnahme zu Hause stattfand. Die Dosis von $300 \mathrm{mg}$ wurde dann weitergegeben.

Am ersten Tag entwickelten 92\% der Kinder Reaktionen vor allem im Be- reich der oberen Atemwege, in der Aufbauphase waren es noch $46 \%$. Die Symptome waren schnell rückläufig. Von den 29 Kindern, die das Protokoll plangemäß beendeten, vertrugen 27 bei der Provokationstestung mit stufenweiser Erhöhung der Dosis bis zu 3,9 g Erdnusseiweiß (kumulativ verabreichte Menge), zwei beendeten die Testung bei 2,1 g.

Sechs Monate nach Beginn der Immuntherapie hatten die Reaktionen auf Erdnuss im titrierten Pricktest ebenso wie die Ergebnisse im Basophilenaktivierungstest signifikant abgenommen. Die Forscher erhöhten nach der Provokationstestung die tägliche Allergendosis weiter bis auf maximal $1.800 \mathrm{mg}$ Erdnussprotein und untersuchten die Kinder weiterhin alle vier Monate. Nach zwölf bis 18

\title{
Anaphylaxie von zwei Seiten in die Zange nehmen
}

\section{Eine Erdnussallergie kann zu anaphylaktischen Reak- tionen führen. Ein doppelter Therapieansatz verspricht zumindest im Tierversuch einen verbesserten Schutz.}

$\mathrm{n}$ einer Studie hatten Forscher mit Hilfe von Erdnussprotein und Choleratoxin Mäuse gegen Erdnuss sensibilisiert. Eine Woche nach der letzten Sensibilisierung erhielten die Tiere vor einem Provokationstest entweder einmalig einen 5-Lipoxigenase-Inhibitor gegen die Leukotrienproduktion, einen Rezeptorantagonisten des Plättchen-aktivierenden Faktors (PAF), ein Antihistaminikum oder kombiniert Antagonisten gegen PAF- und Histaminrezeptor.

Die nur auf Leukotriene oder Histamin abzielende Therapie reduzierte die Schwere und Dauer der anaphylaktischen Reaktion gegenüber unbehandelten Kontrolltieren nicht - die Antagonisierung des PAF-Rezeptors dagegen war wirksam, insbesondere bei der Verhin- derung schwerer Reaktionen. So erholten sich die Mäuse bei PAF-Antagonisierung rascher nach der Provokation: 83\% benötigten für die Normalisierung der Körpertemperatur maximal 120 Minuten. Diese Zeitspanne fiel nur bei $43 \%$ der unbehandelten Mäuse so kurz aus. Eine schwere Hypothermie zwei Stunden nach der Provokation wiesen etwa $30 \%$ der Kontrolltiere, aber nur 9\% der mit dem PAF-Antagonisten behandelten Mäuse auf. Ein Fünftel der unbehandelten Mäuse starb, aber kein Tier in der Gruppe mit PAF-Hemmung.

Die Behandlung mit kombinierter PAF- und Histamin-Antagonisierung verhinderte am besten eine schwere Anaphylaxie: Hier entwickelte nur eine einzige Maus eine schwere systemische
Monaten hatte sich das spezifische $\operatorname{IgE}$ verringert, die $\mathrm{IgG}_{4}$-Konzentration stieg dagegen an.

Innerhalb von sechs bis zwölf Monaten erhöhte sich die Sekretion von Interleukin-(IL) 10, IL-5, Interferon- $\gamma$ und Tumornekrosefaktor- $\alpha$ durch periphere mononukleäre Zellen. T-Zell-Tests zeigten auch eine Herunterregulation verschiedener Apoptosegene, sodass über eine Rolle des programmierten Zelltods bei der Umstimmung des Immunsystems im Rahmen der oralen Immuntherapie spekuliert werden kann.

Fazit: In dieser Studie führte die orale Immuntherapie mit Erdnussprotein zu einer klinisch relevanten Reduktion der allergischen Reaktionen. Auch Veränderungen der humoralen und der zellulären Immunreaktion ließen sich nachweisen.

Jones SM et al. Clinical efficacy and immune regulation with peanut oral immunotherapy. J Allergy Clin Immunol 2009; 124: 292-300 